\title{
Propulsion System Integration and Optimization at the Preliminary Design Phase
}

\author{
$\{\text { Moret, A. Twahir, H. Moustapha, F. Garnier, S. Doré }\}^{*}$, B. Blondin ${ }^{\dagger}$
}

\begin{abstract}
The turbine design of an aero-engine is a multidisciplinary iterative process that involves not only the designing of several components (disc, coverplate, fixing, platform, airfoil, shroud segments, turbine support case, etc.) but also the synergy of multiple disciplines and the application of all their knowledge to create the ideal set of components for the design conditions. Traditionally, the design of these components has been separated into the predetailed and the detailed design phases. Unfortunately during the preliminary stage of the design, engineers are not given enough time to achieve a balance between the fidelity of the results and the time taken to achieve them. This may lead to costly redesign as it is extremely difficult to correct an unsatisfactory concept at a detailed design phase of an engine. The use of Multidisciplinary Design Optimization techniques at a preliminary design phase (Preliminary MDO or PMDO) allows correcting this.

A design system was created that integrates the design of the turbine based on the thermal and mechanical stresses, aerodynamics and the cooling requirements. This was part of an eventual total Propulsion System Integration and Optimization (PSIO) system. Through leveraging of commercial software such as CAD and CAE, parametric models were created for each turbine component and analyzed. This system allows more knowledge to be injected into the early stages of the design process as it helps to rapidly synthesize a turbine and evaluate its attributes over a wide range of alternative designs at a much higher degree of fidelity than was previously possible at the pre-detailed stage. This system also decreases the risk of human errors as it requires less manual inputs than previous pre-detailed processes. This risk is further reduced by the automation of data transfer between the disciplines that would usually be coordinated manually. This automation does not negate the need of engineering judgment but rather more effectively uses an engineer's time by letting him focus only on value added decisions. Finally, the simplifications of the procedures that accompany such a system enable one engineer to be able to design a whole turbine from its components geometric design to the analyses' results post-processing.
\end{abstract}

\section{Introduction}

The design of a gas turbine engine is a multidisciplinary and iterative problem in which the best compromise has to be found between the conflicting disciplines involved: durability, aerodynamics, manufacturing, cost, weight, etc. The design of aero-engines traditionally follows two main stages: preliminary design and detailed design. At the pre-detailed stage, a few groups are involved in the design and analysis of the turbine concept's components and sub-systems; however, the Research and Technology Organization (RTO) of the North Atlantic Treaty Organization (NATO) [1] showed that decisions taken early in the design process are

* École de Technologie Supérieure, Montréal, Québec, Canada

$\dagger \quad$ Pratt and Whitney Canada, Longueuil, Québec, Canada 
often based on low fidelity models and where only little information (data, requirements, etc.) is available. This may compromise the engineers' ability to select the optimal design.

Over the last 40 years or so, significant improvements in computers and computer aided technology $\left(\mathrm{CA}_{\mathrm{x}}\right)$ led to major improvements in many facets of the design and manufacturing of all engineering components. In the analysis world, $\mathrm{CA}_{\mathrm{x}} \mathrm{s}$ such as Finite Element Analysis (FEA) and Computational Fluid Dynamics (CFD) have revolutionized the aerospace industry leading to less testing time and cost as well as limiting the risk of overdesigning and therefore reduced weight as the accuracy of analysis increased. This revolution did, unfortunately, come with its unintended consequences. The more accurate and more complex tools led to an increasing number of specialists and increasingly more specialized departments within companies. Segmenting the Design and Analysis (D\&A) process into smaller and smaller departments with an increasingly narrower and limited mandate led to the 'serial mode of engineering design' [1]. This 'human-centric' approach that accommodated the specialists' understandable need to have autonomy and authority in decision making in their area of expertise led to multiple tools and methodologies being used for sometimes very similar jobs. The isolation of processes, sometimes caused by geographical location differences, meant that there was little communication between the different specialists. The communication between the varied tools used was also lacking as little incentive existed for the specialist to create tools with compatible interfaces, leading to highly coupled complex systems being designed in isolation, at least in the pre-detailed stage. Since large amounts of data is generated by higher fidelity tools, a-lot of human capital is thus expended doing non-value-added tasks such as translation of data from the outputs of one tool to the inputs of another.

A major pitfall of the pre-detailed to detailed design method of designing is that even though knowledge increases during the design process, the freedom to modify any part of the design decreases (Panchencko et al. [2]) as a change has a significant increase in design costs as shown in Fig. 1. Consequently, it is hard to correct a bad concept at a detailed design phase. To correct this, Panchencko et al. [2] suggest the use of MDO at the preliminary design phase, since it is at that stage that the biggest influence on the final product configuration is made. As explained by Martins and Lambe [3], the concept of MDO has been widely studied during the past 50 years. However, there is a lack of information in the literature about using this methodology during the early stages of design. Pachencko et al. [2] explain that an increase of the efforts and knowledge during the pre-detailed design phase implies involving directly the specialist groups instead of waiting until the design phase. This involvement may result in significant delays of the concept evolution since many interactions between several groups are often required. PMDO systems strive to eliminate this short coming as they automate and simplify this iterative process. As written by Pachencko et al. [2], NATO RTO [1] and Korte et al. [4], the following four steps are required to implement a PMDO system:

1. Develop a robust tool base, i.e. design tools based on parametrized CAD models and advanced physics analysis tools;

2. Apply single discipline optimization to individual analytical tools;

3. Create an integration framework, i.e. a software architecture enabling integration, communication and execution of several tools;

4. Implement multidisciplinary optimization with a clear statement of the design objectives, constraints and variables, and an appropriate selection of the algorithms. 


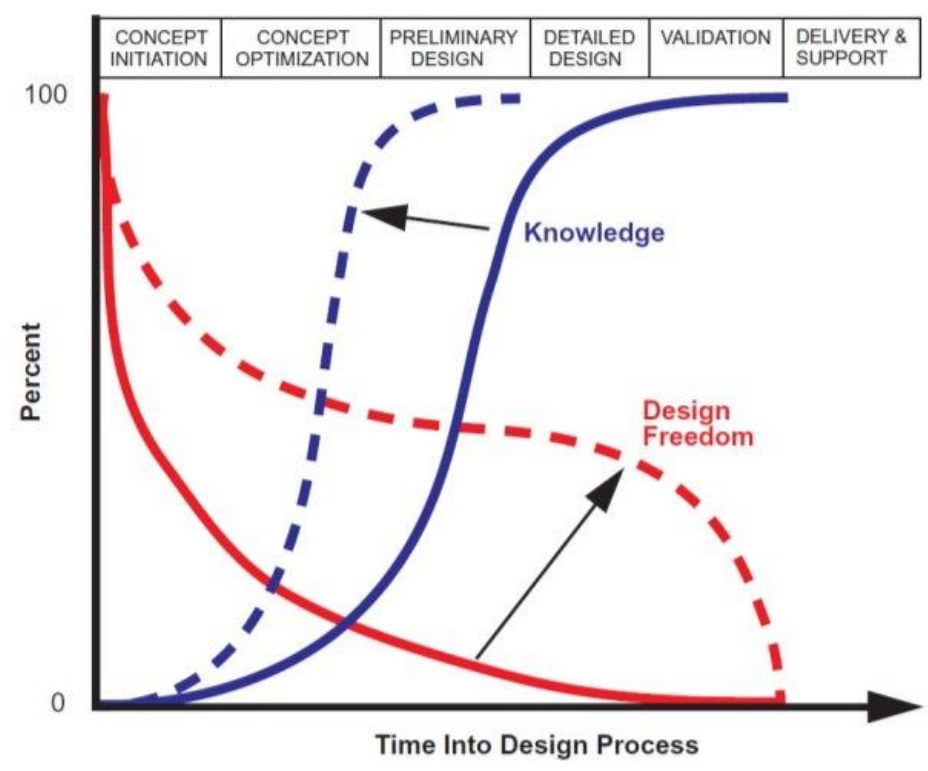

Figure 1: Knowledge vs. design freedom during the design process [1]

A collaborative program was initiated between Pratt \& Whitney Canada (P\&WC) and the École de Technologie Supérieure (ÉTS) to implement an MDO system for designing turbines at the pre-detailed design phase (PMDO). This paper details how these first three steps have been implemented to create the future design system. Some examples of results obtained with this new design system are then presented. The industrial benefits resulting from the implementation are finally outlined.

\section{Development of A Robust Tool Base: Parameterized CAD Models}

The optimization of a turbine rotor, like any other geometry optimization, is crucially dependent on a good modelling of the geometry. The modelling may be in the form of mathematical equations, a set of curves or parametric model; the one common thread is that the model must be "an efficient and flexible description of the geometry" [5]; a geometry model that is able to describe as many real components in the least amount of parameters as possible.

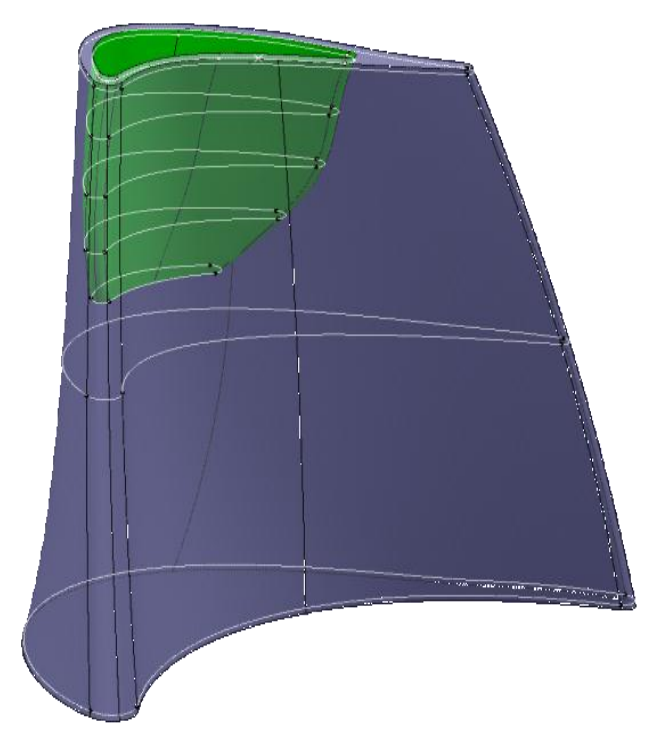

Figure 2: Full 3D Airfoil 
In the modelling of turbine airfoils, many authors use sets of curves to describe the geometry. One such example is the use of Bezier curves in the work of Goel [6] and Tayla et.al. [7]. In some works, a geometry based on company private parametric models have been used. Because of the familiarity of CAD software with most engineers in the field and more important most designers at $\mathrm{P} \& \mathrm{WC}$, in this work parametric models created in CAD software are utilized to give the base of optimization. These models were reported by Lagloire et.al. [8] and Lagloire, Ouellet et.al. [9] (disc geometry), Twahir et al. [10] (fixing geometry) and by Moradi et.al [11] (airfoil geometry shown in Fig. 2). The parametric models will be a basic frame work of the component to be designed that could morph into a vast number of different designs depending on the value assigned to a parameter, very much like how a NACA airfoil could be changed by changing the chord or camber.

\section{Application of A Single Discipline Optimization to Individual Analytical Tools}

Each individual discipline that is used in the design of a turbine requires specific analytical tools, i.e. durability would require Finite Element Analysis (FEA) while Aerodynamics would require Computational Fluid Dynamics (CFD). Each of these analytical tools are computational intensive some taking a few minutes while others a few hours.

During a single optimization run of turbine rotor, the numerical analysis code may be called on dozens if not hundreds of time. The use of a full definition 3D or even quasi 3D analysis all the time may be prohibitive due to the high computational cost and time associated with the multiple runs. It therefore necessitates the use of easy to use, fast approximations of analysis in the running of the initial optimization loops.

\section{Multi-Level Fidelity}

Many techniques exist that assist in the approximation of complex systems but few that are able to properly approximate non-linear, highly coupled, hierarchal problems such as the optimization of a turbine rotor. One such method is the Global reduced - basis approximation where the results are estimated by 'linear Taylor series and the reciprocal Taylor series' [12].

The Variable-Complexity Modelling (VCM) is another means to reduce the computational cost associated with optimization. VCM is a process in which multiple levels of complexity are used in analysis, with each level of complexity calibrated with a level of complexity higher. An example would be the use of simple ' 1 -D' stress analysis that only looks at variation in the stress in one cardinal direction and is then calibrated by the 2-D results (results from the ' $1-\mathrm{D}$ ' are multiplied by a factor equalling previous $2 \mathrm{D} / 1 \mathrm{D}$ results) which in turn are then calibrated by 3-D results. This sort of approximation is used in the design of high-speed civil transport by Hutchison et.al. [13] and also in this work. 1D results shall be calibrated by $\mathrm{K}_{\mathrm{t}}{ }_{1 \rightarrow 2}$ factor (2D results / 1D Results), allowing 1D to predict 2D results just as 2D shall be calibrated by $\mathrm{K}_{\mathrm{t}_{2 \rightarrow 3}}$ factor. The product of these factors will allow 1D results which are much faster to obtain to predict eventual 3D. This is shown in Fig. 3.

With the higher levels of fidelity only used for the calibration of the lower levels of fidelity analysis, it is expected that somewhat of a squared relationship will exist between the uses of the different level of fidelity. Assuming that one can reach the targets within 3 runs, an approximate number of runs is shown in Fig. 8. This thought process would also be used when it comes to obtaining targets. For example, a 3D stress target could be set based on testing and experience and the $K_{t 2 \rightarrow 3}$ factor used to get the $2 \mathrm{D}$ targets. Equally, the $K_{t}{ }_{1 \rightarrow 2}$ factor could then be used to generate 1D targets. 


\section{Approximate number of Runs}

27

9

3

Figure 3: Schematic Representation of Multi-Level Fidelity

Fidelity Level

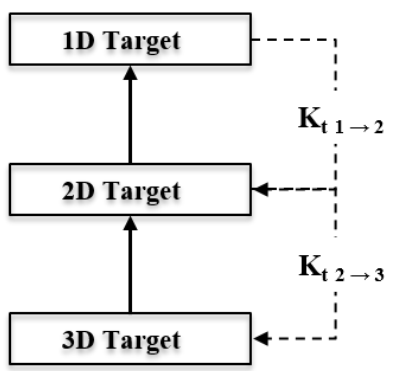

Figure 4: Schematic Representation of Multi-Level Targets

\section{Creation of an Integration Framework}

\section{Modularity}

A high degree of modularity is paramount in a large engineering system as it allows for a large amount of code recycling and greater ease of maintenance. For the work being done, Object Oriented Programming (OOP) is utilized to create a frame that could repeatedly be used throughout the system. Matlab was chosen because of its ease in programming to the average non-software engineer, its debugging features and the documentation features it offers. In order to maintain the component centered approach; a set of functions is to be created that will determine the interaction between all aspects of the system. This modularity will undoubtedly lead to a tool that is much easier to maintain, and will also allow for the creation of a single interface to exist between all modules of PSIO as well as with commercial software.

\section{Data Management}

When integrating vast number of process and methodologies, it is easy to see how a large set of data would need to be transferred. As mentioned by Panchenko et al. [2] the proper management of this data and how it is transferred from one module to another in a systematic way is paramount to the success of the future PSIO design system. The work of NATO RTO [1] recommended that a unique data set should exist in a central location and that each discipline or tool should interact with this data and not have its own data. In this work, the centralized dataset is achieved through the OOP framework outlined previously. The data is stored in one central location and each modules or function is given the reference to that data and not that 
data itself. This eliminated any possibilities of having multiple data of the same parameter, creation a robust and accurate data set. This would also eliminate the non-value-added tasks of transfer of data and the translation of data from the outputs of one tool to the inputs of another.

\section{Examples of Results}

Some work resulting from the new framework outlined has already yielded significant results. This section of the paper summarizes some of them.

\section{Rapid Airfoil 3D (RAF 3D)}

A process that automates the airfoil generation process, referred to as Rapid Airfoil 3D (RAF 3D), was created for uncooled high pressure turbine blades at the preliminary design phase. RAF 3D uses the Turbine Aero Meanline (TAML) in parallel with a database of previously designed $\mathrm{P} \& \mathrm{WC}$ airfoils, in-house design rules and best practices to define a pre-detailed airfoil shape which can be fed back to other analytical groups for pre-detail analyses, such as structures and vibrations.

RAF 3D shortens the turnaround time for P\&WC's turbine aerodynamics group to provide a preliminary 3D airfoil shape to turbine structures group by up to a factor of at least ten. Additionally, the 3D shape will allow other disciplines to assess the characteristics of an actual 3D shape, something that was not necessary available previously at the pre-detailed design stage.

The airfoils generated were found to be competent aerodynamically and when compared to the final detail design was found to be a very good first step. This is shown in figure 5 . When looking at the relative Mach number contours we can see that those are also very similar. This is shown in figure 5 . The stage efficiency of the generated airfoil is only $-0.58 \%$ pts off the detailed design.

\section{High Pressure Turbine Disc Design Space Study}

A parametric disc was created that could model all discs, shown in Figure 6 (a). A Design of Experiment (DOE) was setup to find the parameters that most affect the disc's bore stresses. The DOE showed that the two main design parameters that affect bore stress were the bore width and the bore height (Figure 6 (b)). It also allowed for the creation of design charts that correlated bore stress, burst speed and disc weight to bore width and height.

Figure 7 (a) shows the resulting correlations in 3D surfaces. The projected 2D graph Figure 7 (b), shows the iso-curves for bore stress and weight. The design charts show best ratio between bore width and height to minimize the weight for any given stress value. These design charts offer a better understanding of disc performances within a given design space, and provide help during the generation of a first design by judiciously choosing the initial value of the design parameters.

Finally, as the disc life cycle mostly relies on the bore stress, it is possible to create a design space which quickly guides the preliminary disc design, as shown in Figure 8. The upper limit is defined by the burst optimal line (blue) and the lower by the bore stress (red), on which the disc life relies. This design space is unique to each rotor disc, mostly based on the live rim radius but also on other design parameters. 


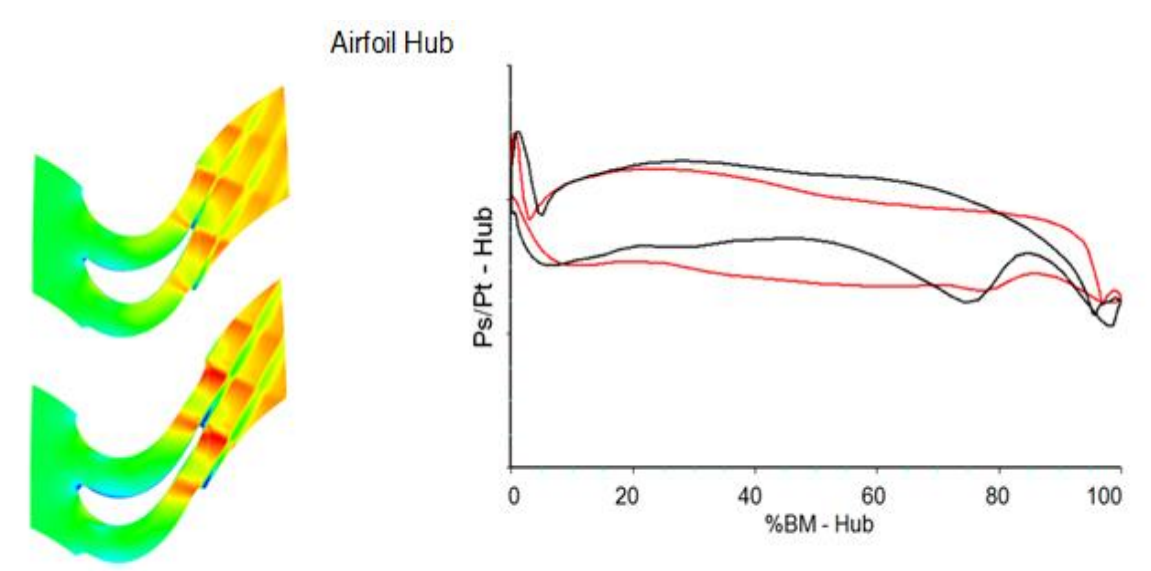

Airfoil Mid
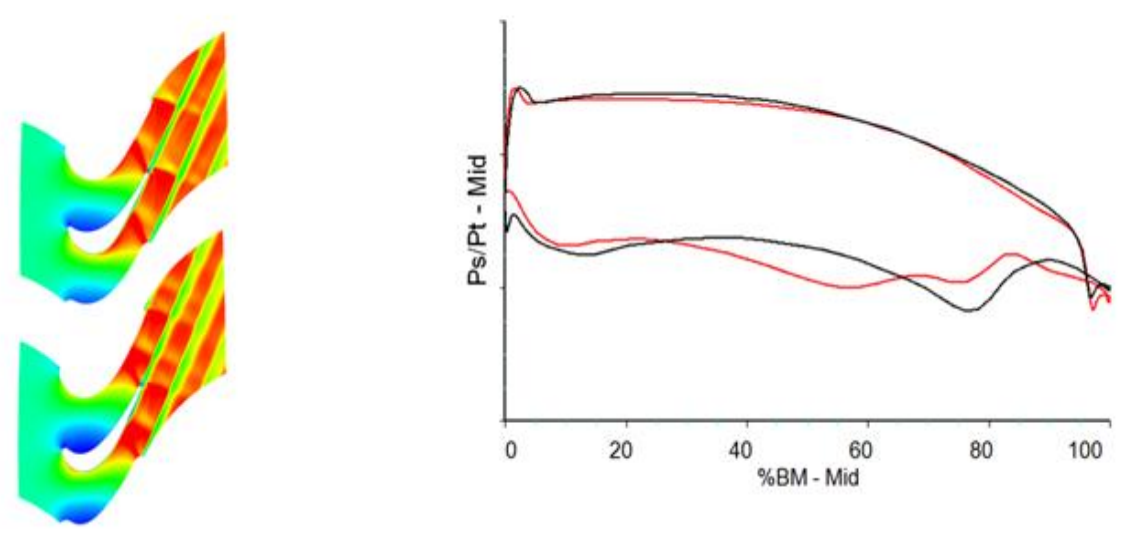

Airfoil Tip
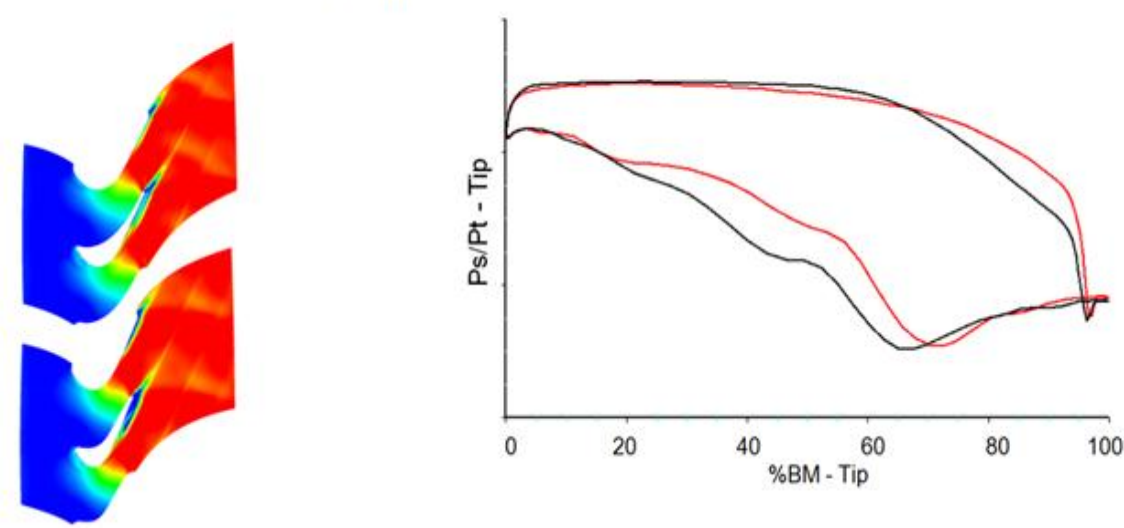

Figure 5 : Test Case I

Left: Airfoil Section Comparison of Final Design (Red) and RAF 3D Concept (Black) Middle: Mnrel Comparison of Final Design (Top) and RAF 3D Concept (Bottom)

Right: Ps/Pt Comparison of Final Design (Red) and RAF 3D Concept (Black) 


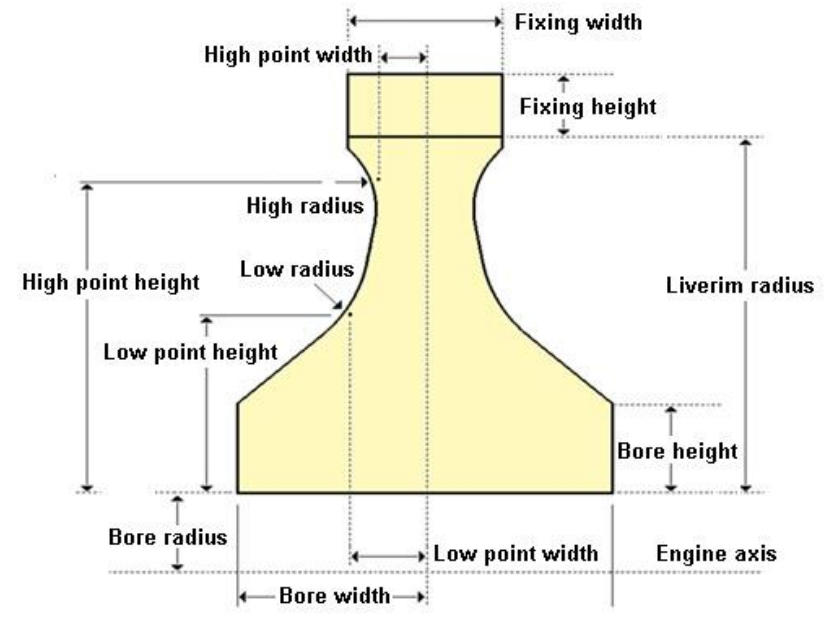

(a)

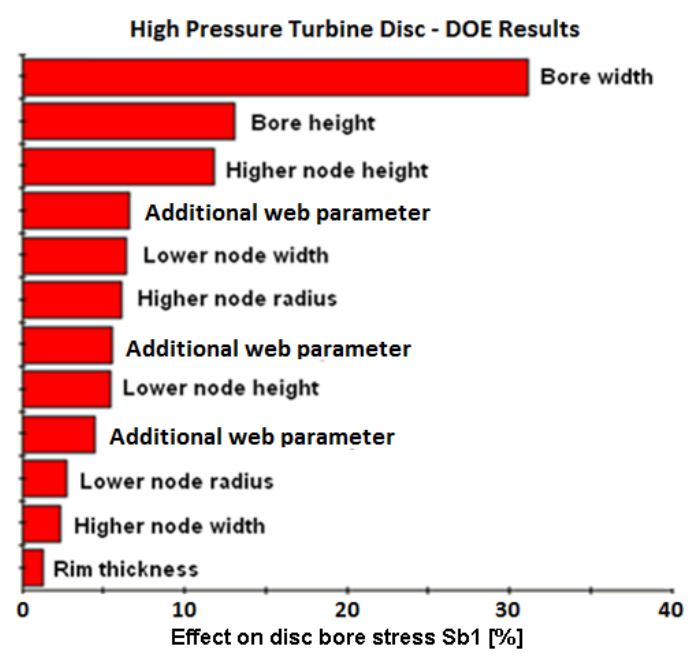

(b)

Figure 6: (a) Disc parametric model, (b) Effect of disc parameters

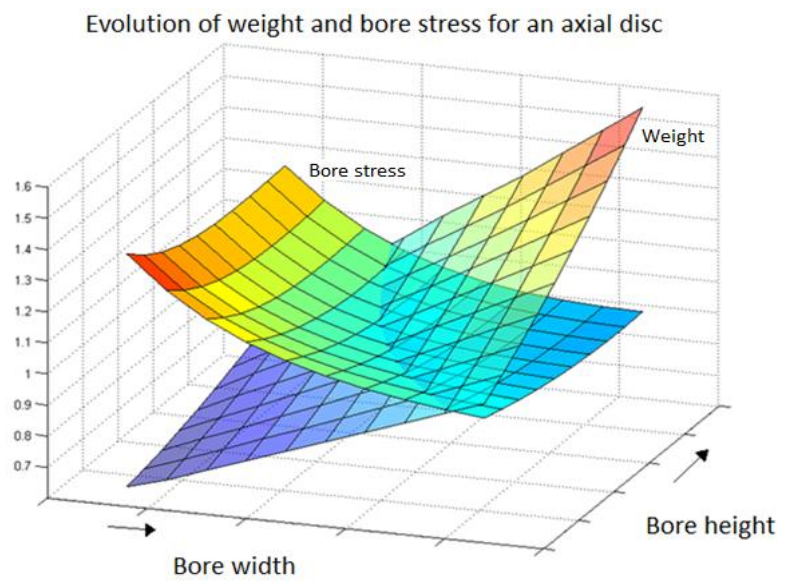

(a)

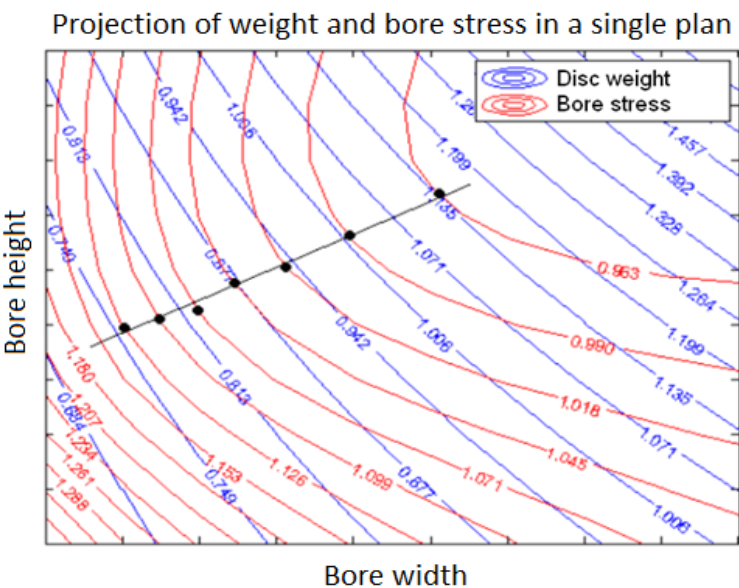

(b)

Figure 7: (a) 3D surfaces of weight and stress, (b) 2D projection of weight and stress

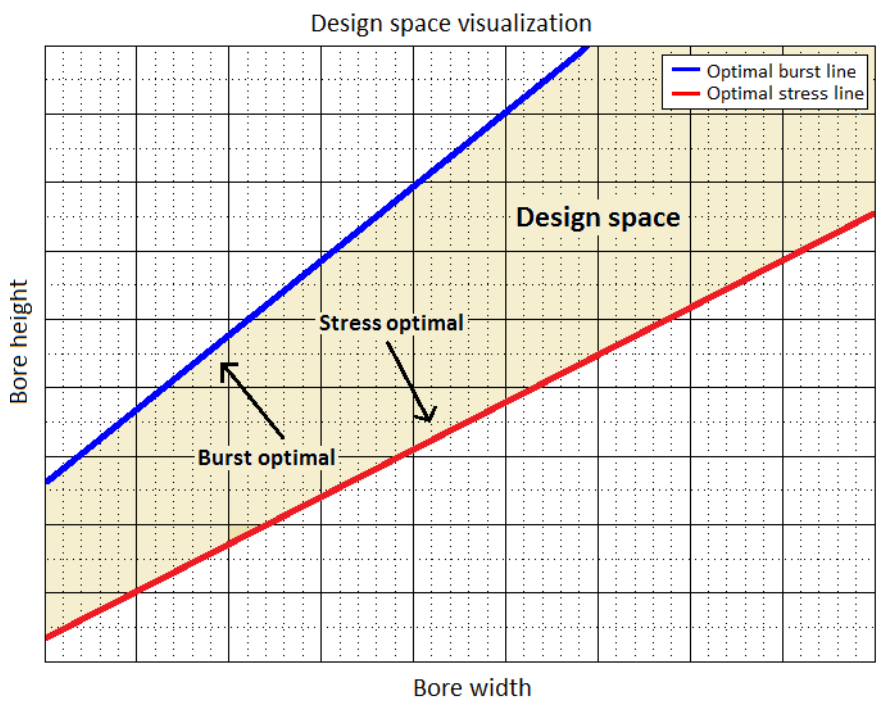

Figure 8: Disc design space 


\section{Turbine Rotor Thermal and Stress Automated Analyses}

To execute a thermal analysis, boundary conditions are required for each specific location (i.e. zone) around the geometry. The Thermal Calculator uses for each zone a specific set of inputs obtained from all the connected sub-systems (performance data, design CAD sub-systems, a Secondary Air System generator, etc.) to calculate the thermal boundary conditions. Thanks to a Gateway software, the Rotor Analysis FEA sub-system is able to make use of the CAD parametric models introduced in this paper to identify easily each zone of the rotor geometry. Thermal boundary conditions correspond to a combination of a heat transfer coefficient and a bulk temperature at the surface of each zone. The real challenge here was therefore to select the proper correlations for the heat transfer coefficients considering that these are directly influenced by the zone's geometry and the physic at stake. At a preliminary design stage, the zones around the rotor can be modelled using basic cases such as flat plates, rotating disks, channels, etc. An example of the choices made are represented in Figure 9.

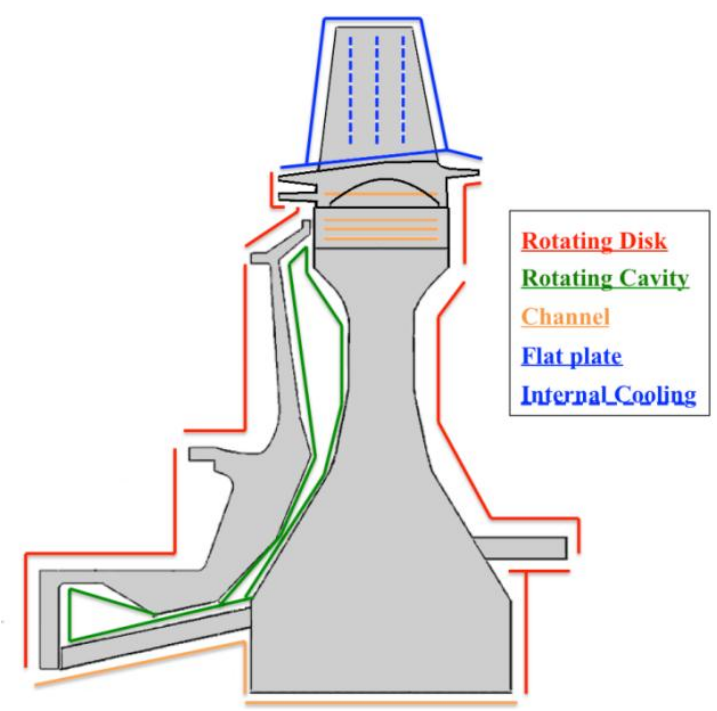

Figure 9: Rotor HTC zones

A program was developed in the FEA software in order to automate the analyses process. Depending on the configuration of the studied rotor, this system loads the different components' 2D geometry, or the cross section of the 3D components, from the CAD models. The loaded geometries are assembled and meshed using the appropriate elements. The calculated thermal boundary conditions, varying in time in accordance with the mission, are applied on the zones identified in the FEA program. For the stress analysis, boundary and load conditions are much simpler to evaluate and apply considering that they consist of fixing the disk's bore to prevent any radial translation, using the thermal results and applying the proper rotational speed. Centrifugal forces are easily computed by combining the elements' mass matrices and the rotational velocities.

Thermal and stress analyses were carried out for one turbine stage of three different engines. Those engines are of different configurations: a turboshaft as test 1, a high-bypass ratio turbofan as test 2 and a turboprop as test 3 , and different turbine stages were chosen: two high pressure turbines and one low pressure turbine. Figure 10 (a) and Figure 10 (b) show an example of results obtained for a thermal analysis done on the test case number 2. On the Figure 10 (b), one can observe the evolution of the temperature probed at five key locations around the rotor represented on the temperature contours of Figure 10a. As absolute values cannot be disclosed for intellectual property reasons, non-dimensionalized scales where used in Figure 10 (a) and Figure $10(b)$. 


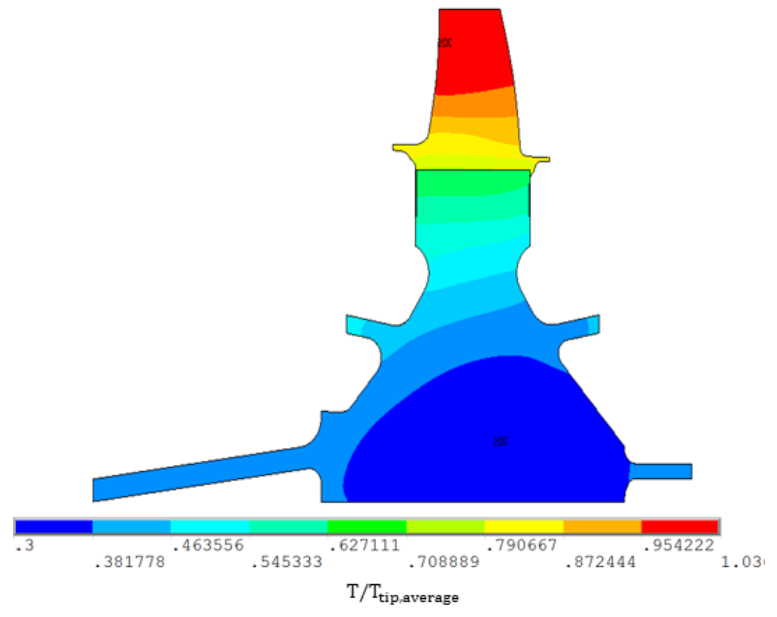

(a)

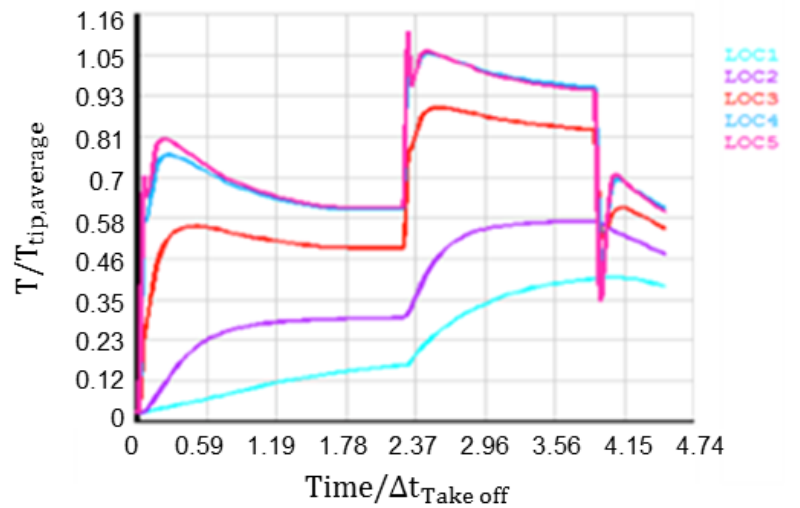

(b)

Figure 10: Thermal analysis results example performed on test case 2: (a) contour plot, (b) transient plot of temperatures at key locations

\section{Industrial Benefits}

\section{Productivity}

The time required to execute these analyses was compared to what is usually taken by a P\&WC expert using the existing pre-detailed design process. Although bringing more knowledge in the early stages of the design process usually increases the time spent at the pre-detailed stage, it was found that through the integration of the various disciplines involved in the design and analysis of a turbine's rotor, and due to the automation and simplification of the process (data automatic extraction/generation, reduction of the number of inputs required from the user, etc.), the time to get a first pass of results was reduced by $80 \%$. It was indeed evaluated that could now be obtained in days what was usually the result of weeks of work.

In the future, it is expected that with more accurate preliminary results comes a reduction of the number of iterations required at the detailed design phase. It was estimated by several specialists that the detailed design process could be shortened by an entire design cycle. Considering that a detailed design process usually requires around three passes of the design cycle, the system introduced in this work could lead to a time reduction of about $30 \%$ on the whole rotor detailed design phase. This estimation was made on the grounds that a more accurate pre-detailed design, obtained by taking into account more data (i.e. parameterized models, secondary air system, thermals, and even more disciplines incorporated in the other modules), will remove the need for a first pass in the detailed design phase to generate the missing data of a conventional preliminary process. 


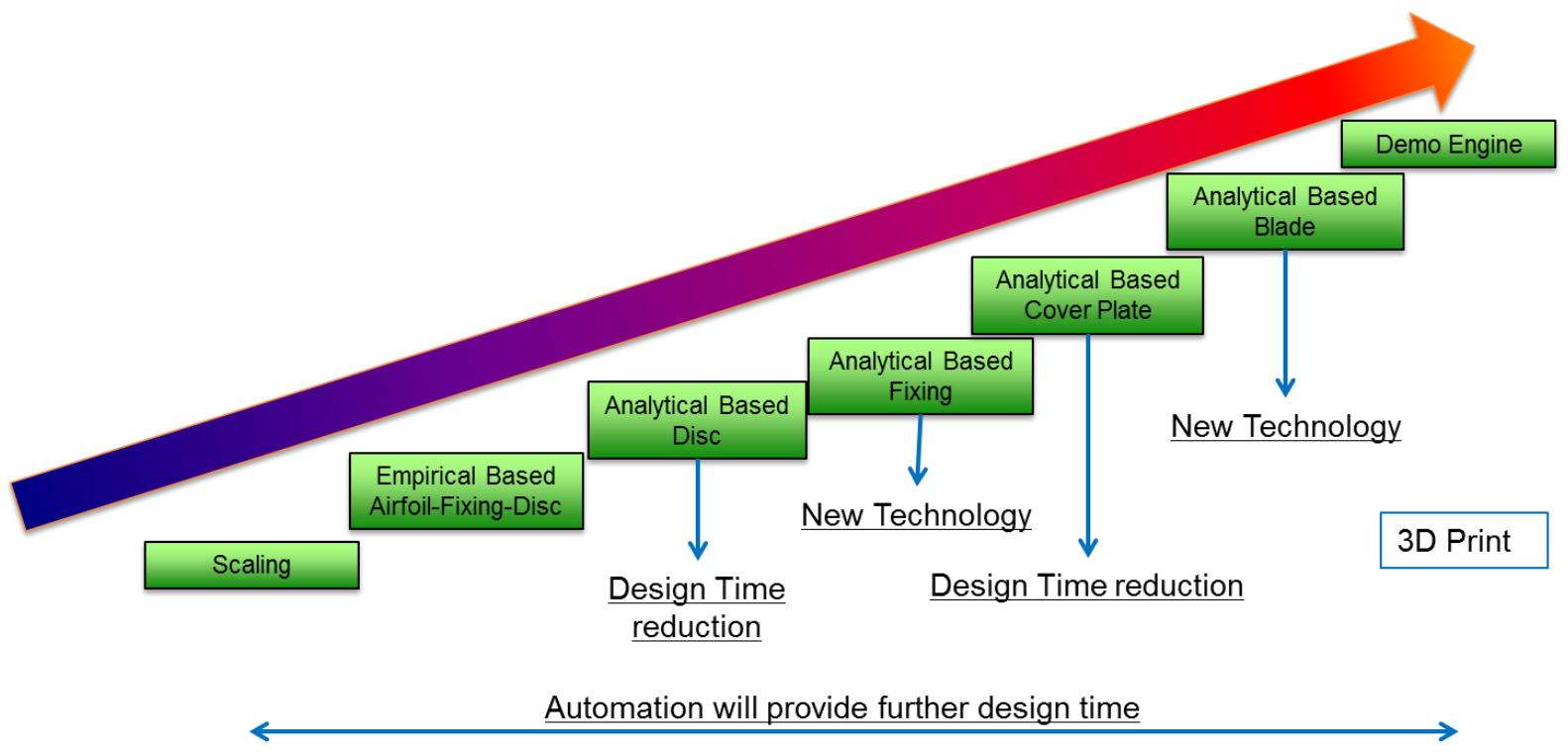

Figure 11: PSIO provides an increased level of knowledge as well as reducing time taken

\section{Quality}

The use of CAD software to provide more accurate area data, has also led to an increase in the fidelity of the 1D result. Before PSIO the pre-detailed of rotors would rely on established rules of thumbs in conjunction with 1D analysis. The creation and injection of a 2D design methodology into the pre-detailed design process of some components has greatly increased the fidelity of the analysis, in some cases an almost $100 \%$ increase in the fidelity was obtained, this allows the quality of the results to reach a theoretical limit. This decrease is shown in Figure 12.

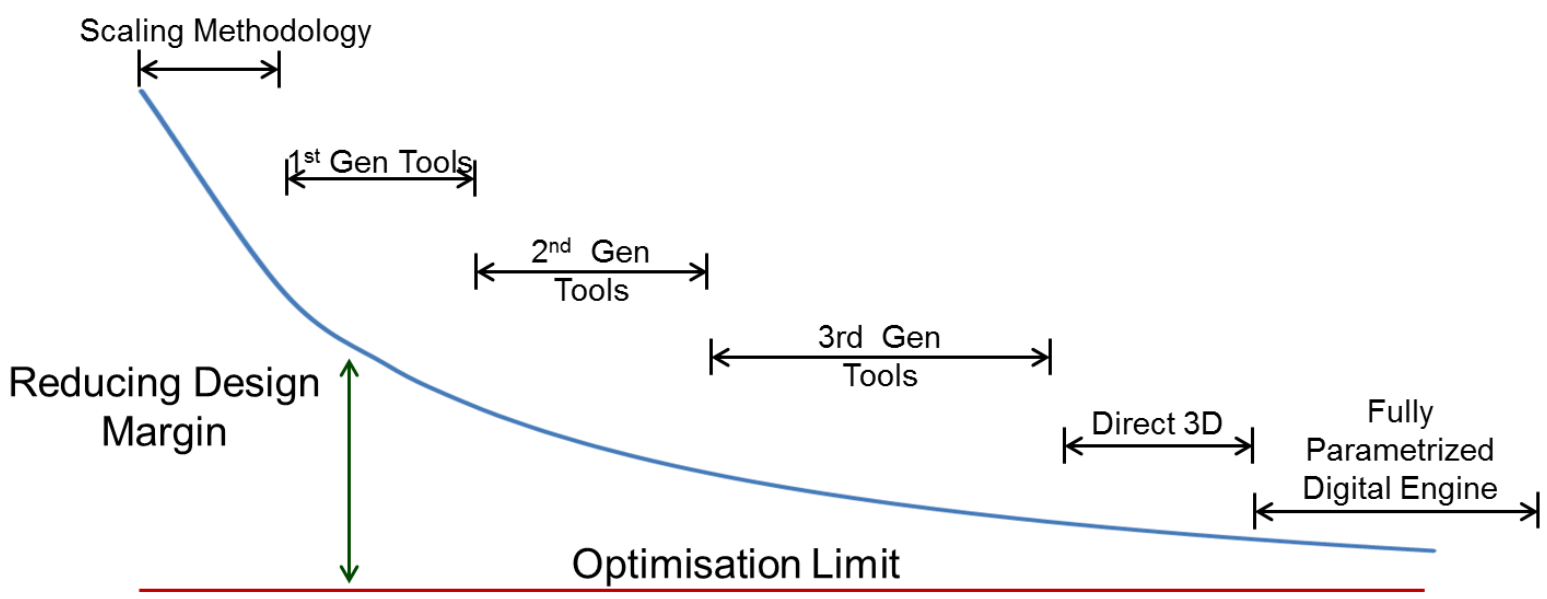

Figure 12 Decrease in the Quality between the tools and theoretical limit

At the preliminary design phase of a turbine design, some analyses are usually not executed. It is the case for the rotor thermal analyses which are instead replaced by scaled temperature maps based on legacy data. Getting the right scaling factors can be a long and tricky process, and the 
temperatures so obtained are often found to be off once in detailed design phase. Considering that, one understands that comparing the results obtained in the rotor thermal analysis portion of this work with pre-detailed level results is complicated. However, it is generally accepted that pre-detailed design results are about $30 \%$ off. Figure 13 represents the absolute average difference between the transient temperatures obtained with the rotor thermal analysis system described previously and with detailed design level tools at various locations around each of the three turbine's rotor. Test 1 is an engine with a cover-plate explaining why seven (instead of five) locations are present for that test case. A maximum difference of $7 \%$ was obtained for one test case at one location. That result is explained by the simplified geometry compare to a detailed final design (definition and size of the holes, channels and other features for example), which has an impact on both the secondary air system and the thermal boundary conditions. The second source of difference with the detail design process is the simplicity of the models used for the calculation of the heat transfer coefficients and the number of zones used. However, a difference of less than $7 \%$ at the pre-detailed level compared to detailed design results is excellent Moreover, the average of the differences of the three turbines at all the locations is $2.72 \%$ with a standard deviation of $2.13 \%$.

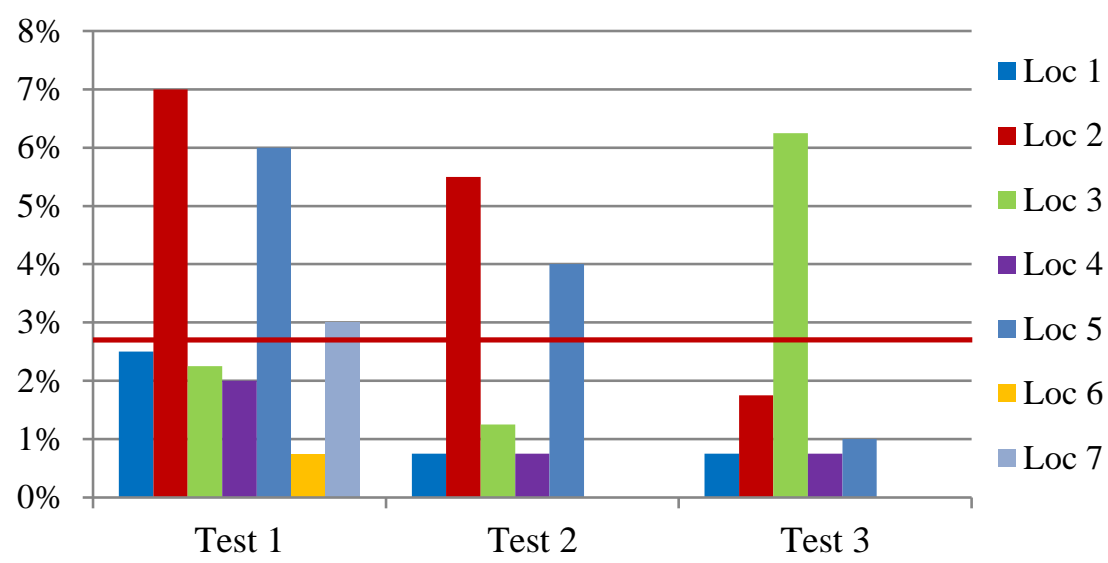

Figure 13: Presented work vs. Detailed Design results

\section{Improved Resource Allocation}

One of the greatest resources in any company is the time of the highly experienced engineers. The new design system will allow the engineers to spend less time transferring data from one specialist owned software to the other and worrying about its accuracy, to focus on designing more and better components. This will allow them to focus most of their time on value added tasks rather than mundane ones (open/close software products, copy/paste data files).

Another consequence is that the CAx software licenses that are usually in such high demand will become more readily available. This is because the new system only uses the licenses for the short duration they need them to transfer data (less than 10 seconds) or when the FEA is running (less than 5 minutes) while currently a user could take much longer than that to perform the tasks manually.

This work confirmed that an automation of the preliminary design process is possible. The results presented in this paper suggest that one engineer could be in charge of designing and analysing an entire rotor during the pre-detailed phase, without the need for many specialized groups to be involved.

Moreover, by requiring fewer user inputs and removing the non-value added task for an engineer to manage data, this system decreases the risk of human errors while entirely leaving the important decisions to the user. 


\section{Conclusion and Recommendation}

This paper presented an improved and integrated design system that would greatly aid in the design and analysis of turbine rotors at the pre-detailed design phase.

The tool developed enables a cohesive single integrated simulation environment capturing the strength of targeted commercial software for their specific capabilities. Speed and higher fidelity, brought about by FEA and CFD, are two key features that make a better design system for the preliminary design phase.

The two biggest impacts the proposed PMDO new design system will have are in improved productivity and improved quality of results when compared to previous pre-detailed design tools.

The framework created, although done for the pre-detailed phase of the design of rotors, could be used for the detailed design phase. The lessons learnt on how to share data, incorporate different disciplines and design methodology could be injected into the detailed design stage. By using the same framework and changing the individual analysis tools used (CFD and FEA) through increasing number of nodes or by making less simplification would increase the accuracy of results to the level required at that stage of design. Changing the underlying parametrized $\mathrm{CAD}$ models would offer the engineer much greater flexibility to adjust the geometry to the most granular level.

This framework, with a few adjustments, could also be used for other parts of an engine with rotors, i.e. compressors. Although the analysis tools and parametrized models might change, the core, in terms of design system, multi-level fidelity tools, the interfaces created to commercial software, the lessons learnt in data management and progress monitoring could be easily transferred into creating a PMDO design system for compressors.

\section{References}

[1] NATO Science and Technology Organization. (2006). Integration of Tools and Processes for Affordable Vehicles. Chapter 3: Air Vehicles. NATO RTO Research Task Group AVT 093.

[2] Panchenko, Y., Patel, K., Moustapha, H., Dowhan, M. J., Mah, S., \& Hall, D. (2002). Preliminary Multi-Disciplinary Optimization in Turbomachinery Design. Proceedings of RTO/AVT symposium on "Reduction of Military Vehicle Acquisition Time and Cost through Advanced Modelling and Virtual Simulation", (p. 22). Paris, France: RTO-MP089.

[3] Martins, J. R., \& Lambe, A. B. (2013). Multidisciplinary Design Optimization: A Survey of Architectures. AIAA Journal, Vol. 51(No. 9), 2049-2075.

[4] Korte, J. J., Weston, R. P., \& Zang, T. A. (1998). Multidisciplinary Optimization Methods for Preliminary Design. Multidisciplinary Optimization Branch, MS 159, NASA Langley Research Center.

[5] Sobieszczanski-Sobieski, Jaroslaw and Haftka, Raphael, 1997,"Multidisciplinary aerospace design optimization: survey of recent developments", Structural optimization 14.1, pp. 1-23.

[6] Goel, S., "Turbine Airfoil Optimization Using Quasi-3D Analysis Codes", International Journal of Aerospace Engineering, vol. 2009.

[7] Tayla, S., Chattopadhyay, A., and Rajadas, J., 2002,"Multidisciplinary Design Optimization Procedure for Improved Design of a Cooled Gas Turbine Blade", Engineering Optimization, vol. 34(2), pp. 175-194. 
[8] Ouellet, Y., Garnier, F., Roy, F., and Moustapha, H., 2014, "A Preliminary Design System for Turbine Discs", in ASME Turbo Expo 2014: Turbine Technical Conference and Exposition, vol. 2B, Düsseldorf, Germany.

[9] Lagloire, F. and Moustapha, H., 2013, "Single Platform Integration Environment for Turbine Rotor Design \& Analysis", in American Institute of Aeronautics and Astronautics, Busan, Korea.

[10] Twahir, A., Roy, F., Moustapha, H., and Attia, M., 2014, "Preliminary Design and Analysis Tool For Aeroengine Turbine Fixings", in ASME, vol. 1, Montréal.

[11] Moradi, N., Vlasic, E., and Moustapha, H., 2015, "Rapid Airfoil Design for Uncooled High Pressure Turbine Blades", in ASME Turbo Expo 2015: Turbine Technical Conference and Exposition, vol. 2A, Montreal.

[12] Jha, R., 1999, "Development of Multidisciplinary Design Optimization For Smart Composit Wings And Turbomachinery Blades".

[13] Hutchison, M., Unger, E., Mason, W., Grossman, B., and Haftka, R., "Variablecomplexity aerodynamic optimization of a high-speed civil transport wing", Journal of Aircraft, pp. 110-116. 